\title{
DATABASES ANALYSIS OF HYDROPOWER RESOURCE Potential AROUND THE GLOBE
}

\author{
Qasim Rauf, YanpinLi and Anam Ashraf \\ North China University of Water Resources and Electric Power, Henan, China
}

\begin{abstract}
The renewable generation is one of the fast growing power system. Whereas the world is facing the challenge of effectively exploiting and utilizing renewable energy resources, not only to meet the increasing energy demand, but also to preserve and to reduce the depletion of fossil fuels and to lessen the amount of $\mathrm{CO} 2$ emissions in our atmosphere. The national energy generation resources of every country plays an important role in the development. The energy transition is well underway in most European countries. It has a growing impact on electric power systems as it dramatically modifies the way electricity is produced. In this paper, our focus is to perform a systematic review of hydropower resource potential around the Globe. In this study we will address three research queries: 1) what is the existing status of hydropower resource potential around the globe? 2) What kind of methodologies frameworks and approaches are used for exploiting and utilizing renewable energy resources 3) What are the limitations of exploiting renewable resource potentials. The purpose of the study is to highlight the current research issues, to provide valid solutions to these issues and to find out the limitations of existing work in this area of hydropower resource potential. This will be done by performing quantitative literature analysis of different databases and all the results will be gathered by analysing the statistical data using "SPSS". Remedial techniques for handling the limitation of usability engineering management will be planned in future.
\end{abstract}

\section{KEYWORDS}

Sustainable energy resource; Hydroelectricity; Renewable energy source; Sustainable hydropower; Hydroelectric energy; Hydropower technology ; Hydroelectric power Hydropower; Resource potential; Systematic study of hydropower; Database analysis of resource potential; Resource potential around the globe

\section{INTRODUCTION}

Lack of latest power generation techniques leads to unavailability of electricity to the population and they depend on traditional systems. According to the study, it was revealed that more than 1.27 billion people worldwide do not have access to electricity in 2010 [1]. Almost all countries around the world have recognized the importance of dominance which is to be self-sufficient for power generation and management. To deal with the universal scatter gap, the power system must undergo an insurgency that specializes in learning about the transition from fossil fuels to renewable energy. Long ago, meeting the electricity demand through adequate supply of electricity was a major problem. This is a major task for renovating the power network that is responsible for the supply, because of the extraordinary inadequacies, the inability of management to cope with the backing mechanism that leads to serious issues of "circular debt", land imbalances Near recovery and impractical electrical costs [2]]. Energy consumption has grown rapidly in the last decade globally and is projected to increase by $33 \%$ from 2010 to 2030 [3]. Lack of fossil fuel resources is a major threat to the world economy and is a factor. Today the world faces the most important economic, environmental and developmental issues. The energy sector accounts for $75 \%$ of global GHG emissions [4]. The steady increase in global energy demand has led to an increase in carbon dioxide ( $\mathrm{CO} 2)$ emissions and is a serious impact 
to the environment and a significant contributor to global climate change. The relationship between renewable and non-renewable energy sources, economic development, and $\mathrm{CO} 2$ emissions has been investigated in several studies. Many people have used panel country data to examine these relationships. For example, Apergis and Payne [5] studied 80 countries and found bidirectional causalities between renewable energy consumption and economic growth and between non-renewable energy consumption and economic growth. Hydroelectric power generation contributes about $16 \%$ of global electricity generation [6]. In 2010 it produced threefourths of all global renewable electricity. Large and small hydro contributed $70 \%$ and $6 \%$ with half the production coming from China, Brazil, United States, Canada and Russia respectively. In some countries, including Brazil and many African countries, $75 \%$ of grid electricity comes from hydro.

In study, systematic review research questions are worked by the "PICOC" structure against research question look into strings are worked for various query strings including various databases ,"IEEE, ACM", “GOOGLE SCHOLAR" and "SCIENCE DIRECT". Databases are made against each query strings and query protocol is implemented on the databases for conclusive assurance of papers, with assistance of "data extraction frames" information from each chosen paper is extricated and checked on statistically

\section{Protocol for Search Process}

Consequent to deciding inquire about questions

a survey convention is created which consolidates the accompanying:

- The Search Process

- "Inclusion" \& "Exclusion" Criteria

- The Selection Procedure

- The Data Extraction Process

- Data Synthesis

\section{SOURCES FOR Literature SEARCH}

Hydropower resource potential is a wide and intrigue field with conflicting terminologies. We searched "Springer", "IEEE", "Science Direct" and "ACM "for primary studies

\section{Literature SEARCh STRATEgY}

\subsection{Research Question in PICOC Structure}

I. RQ1: What is the existing status of hydropower resource potential around the globe?

- Population: Citizens

- Intervention: Hydropower resource Potential management

- Outcome: Status of Hydropower Resource Potential around the globe

\section{1) Search Strings/Second Step: Synonyms}

\section{a) Population}

\section{"Citizens}




\section{b) Intervention}

"Resource potential Management", "Hydropower resource potential management approaches" , "Hydropower resource potential Evaluation", "Hydropower resource potential development approaches" "Hydropower resource potential development techniques"

\section{c) Outcome}

"Hydropower trends around the Globe" , "Resource Potential Status", "Status of Resource Potential Management", "Current Resource Potential Status", "Hydropower Resource Potential status", "Hydropower Resource Potential Trends and Status"

\section{2) Strings Used for Essential "Primary" Studies Search of Research Question 1}

Table 1: Strings used for primary studies search of the existing status of hydropower resource potential around the globe

\begin{tabular}{|c|c|}
\hline Database & Search String \\
\hline Springer & $\begin{array}{l}\text { "Resource potential Management", "Hydropower resource potential management } \\
\text { approaches", "Hydropower resource potential Evaluation", "Hydropower resource } \\
\text { potential development approaches" "Hydropower resource potential development } \\
\text { techniques", "Hydropower resource potential measurement", "Resource Potential", } \\
\text { "Hydropower resource potential Analysis", "Resource potential management } \\
\text { methodologies", "Hydropower resource potential methodologies", "Resource potential } \\
\text { Essentials", "Resource potential methods", "Resource potential development processes", } \\
\text { "Resource potential practices", "Resource potential approaches", Hydropower resource } \\
\text { potential techniques" }\end{array}$ \\
\hline$A C M$ & $\begin{array}{l}\text { "Resource potential Management", "Hydropower resource potential management } \\
\text { approaches", "Hydropower resource potential Evaluation", "Hydropower resource } \\
\text { potential development approaches" "Hydropower resource, potential development } \\
\text { techniques", "Hydropower resource potential measurement", "Resource Potential", } \\
\text { "Hydropower resource potential A Analysis", "Resource, potential management } \\
\text { methodologies", "Hydropower resource potential methodologies", "Resource potential } \\
\text { Essentials", "Resource potential methods", "Resource potential development processes", } \\
\text { "Resource potential practices", "Resource potential approaches", Hydropower resource } \\
\text { potential techniques". }\end{array}$ \\
\hline IEEEE & $\begin{array}{l}\text { "Resource potential Management", "Hydropower resource potential management } \\
\text { approaches", "Hydropower resource potential Evaluation", "Hydropower resource } \\
\text { potential development approaches" "Hydropower resource, potential development } \\
\text { techniques", "Hydropower resource potential measurement", "Resource Potential", } \\
\text { "Hydropower resource potential Analysis", "Resource potential management } \\
\text { methodologies", "Hydropower resource potential methodologies", "Resource potential } \\
\text { Essentials", "Resource potential methods", "Resource potential development processes", } \\
\text { "Resource potential practices", "Resource potential approaches", Hydropower resource } \\
\text { potential techniques". }\end{array}$ \\
\hline $\begin{array}{l}\text { Science } \\
\text { Direct }\end{array}$ & $\begin{array}{l}\text { "Resource potential Management", "Hydropower resource potential management } \\
\text { approaches", "Hydropower resource potential Evaluation", "Hydropower resource } \\
\text { potential development approaches" "Hydropower resource, potential development } \\
\text { techniques", "Hydropower resource potential measurement", "Resource Potential", } \\
\text { "Hydropower resource potential Analysis", "Resource potential management } \\
\text { methodologies", "Hydropower resource potential methodologies", "Resource potential } \\
\text { Essentials", "Resource potential methods", "Resource potential development processes", } \\
\text { "Resource potential practices", "Resource potential approaches", Hydropower resource } \\
\text { potential techniques". }\end{array}$ \\
\hline
\end{tabular}

II. RQ2: What kind of methodologies frameworks and approaches are used for exploiting and utilizing renewable energy resources

\section{1) Search Strings/Second Step: Synonyms}

\section{a) Population}

\section{Citizens}




\section{b) Intervention}

"Resource potential Management", "Hydropower resource potential management approaches", "Hydropower resource potential Evaluation", "Hydropower resource potential development approaches" "Hydropower resource potential development techniques"

\section{c) Outcome}

"Hydropower trends around the Globe", "Resource Potential Status", "Status of Resource Potential Management", "Current Resource Potential Status", "Hydropower Resource Potential status", "Hydropower Resource Potential Trends and Status"

\section{2) Strings Used for Essential “ Primary” Studies Search of Research Question 2}

Table 2: What kind of methodologies frameworks and approaches are used for exploiting and utilizing renewable energy resources

\begin{tabular}{|c|c|}
\hline Database & Search String \\
\hline Springer & $\begin{array}{l}\text { "Resource potential Management", "Hydropower resource potential management } \\
\text { approaches", "Hydropower resource potential Evaluation", "Hydropower resource } \\
\text { potential development approaches" "Hydropower resource, potential development } \\
\text { techniques", "Hydropower resource potential measurement", "Resource Potential", } \\
\text { "Hydropower resource potential Analysis", "Resource potential management } \\
\text { methodologies", "Hydropower resource potential methodologies", "Resource potential } \\
\text { Essentials", "Resource potential methods", "Resource potential development processes", } \\
\text { "Resource potential practices", "Resource potential approaches", Hydropower resource } \\
\text { potential techniques" }\end{array}$ \\
\hline$A C M$ & $\begin{array}{l}\text { "Resource potential Management", "Hydropower resource potential management } \\
\text { approaches", "Hydropower resource potential Evaluation", "Hydropower resource } \\
\text { potential development approaches" "Hydropower resource, potential development } \\
\text { techniques", "Hydropower resource potential measurement", "Resource Potential", } \\
\text { "Hydropower resource potential Analysis", "Resource, potential management } \\
\text { methodologies", "Hydropower resource potential methodologies", "Resource potential } \\
\text { Essentials", "Resource potential methods", "Resource potential development processes", } \\
\text { "Resource potential practices", "Resource potential approaches", Hydropower resource } \\
\text { potential techniques". }\end{array}$ \\
\hline IEEE & $\begin{array}{l}\text { "Resource potential Management", "Hydropower resource potential management } \\
\text { approaches", "Hydropower resource potential Evaluation", "Hydropower resource } \\
\text { potential development approaches" "Hydropower resource, potential development } \\
\text { techniques", "Hydropower resource potential measurement", "Resource Potential", } \\
\text { "Hydropower resource potential Analysis", "Resource potential management } \\
\text { methodologies", "Hydropower resource potential methodologies", "Resource potential } \\
\text { Essentials", "Resource potential methods", "Resource potential development processes", } \\
\text { "Resource potential practices", "Resource potential approaches", Hydropower resource } \\
\text { potential techniques". }\end{array}$ \\
\hline $\begin{array}{l}\text { Science } \\
\text { Direct }\end{array}$ & $\begin{array}{l}\text { "Resource potential Management", "Hydropower resource potential management } \\
\text { approaches", "Hydropower resource potential Evaluation", "Hydropower resource } \\
\text { potential development approaches" "Hydropower resource, potential development } \\
\text { techniques", "Hydropower resource potential measurement", "Resource Potential", } \\
\text { "Hydropower resource potential Analysis", "Resource potential management } \\
\text { methodologies", "Hydropower resource potential methodologies", "Resource potential } \\
\text { Essentials", "Resource potential methods", "Resource potential development processes", } \\
\text { "Resource potential practices", "Resource potential approaches", Hydropower resource } \\
\text { potential techniques". }\end{array}$ \\
\hline
\end{tabular}

III. RQ3: What are the limitations of exploiting renewable resource potentials?

\section{1) Search strings/Second Step :Synonyms}

\section{a) Population}

\section{"Citizens"}

\section{b) Intervention}

"Resource potential Management", "Hydropower resource potential management approaches", "Hydropower resource potential Evaluation", "Hydropower resource potential development approaches" "Hydropower resource potential development techniques" 


\section{c) Outcome}

"Hydropower trends around the Globe" , "Resource Potential Status", "Status of Resource Potential Management", "Current Resource Potential Status", "Hydropower Resource Potential status", "Hydropower Resource Potential Trends and Status"

\section{1) Strings Used for Primary Studies Search of Research question 3}

Table 3: What are the limitations of exploiting renewable resource potentials?

\begin{tabular}{|c|c|}
\hline Database & Search String \\
\hline Springer & $\begin{array}{l}\text { "Resource potential Management", "Hydropower resource potential management } \\
\text { approaches", "Hydropower resource potential Evaluation", "Hydropower resource } \\
\text { potential development approaches" "Hydropower resource, potential development } \\
\text { techniques", "Hydropower resource potential measurement", "Resource Potential", } \\
\text { "Hydropower resource potential Analysis", "Resource potential management } \\
\text { methodologies", "Hydropower resource potential methodologies", "Resource potential } \\
\text { Essentials", "Resource potential methods", "Resource potential development processes", } \\
\text { "Resource potential practices", "Resource potential approaches", Hydropower resource } \\
\text { potential techniques" }\end{array}$ \\
\hline$A C M$ & $\begin{array}{l}\text { "Resource,potential Management", "Hydropower resource potential management } \\
\text { approaches", "Hydropower resource potential Evaluation", "Hydropower resource } \\
\text { potential development approaches" "Hydropower resource, potential development } \\
\text { techniques", "Hydropower resource potential measurement", "Resource Potential", } \\
\text { "Hydropower resource potential A Analysis", "Resource, potential management } \\
\text { methodologies", "Hydropower resource potential methodologies", "Resource potential } \\
\text { Essentials", "Resource potential methods", "Resource potential development processes", } \\
\text { "Resource potential practices", "Resource potential approaches", Hydropower resource } \\
\text { potential techniques". }\end{array}$ \\
\hline IEEE & $\begin{array}{l}\text { "Resource,potential Management", "Hydropower resource potential management } \\
\text { approaches", "Hydropower resource potential Evaluation", "Hydropower resource } \\
\text { potential development approaches" "Hydropower resource, potential development } \\
\text { techniques", "Hydropower resource potential measurement", "Resource Potential", } \\
\text { "Hydropower resource potential Analysis", "Resource, potential management } \\
\text { methodologies", "Hydropower resource potential methodologies", "Resource potential } \\
\text { Essentials", "Resource potential methods", "Resource potential development processes", } \\
\text { "Resource potential practices", "Resource potential approaches", Hydropower resource } \\
\text { potential techniques". }\end{array}$ \\
\hline $\begin{array}{l}\text { Science } \\
\text { Direct }\end{array}$ & $\begin{array}{l}\text { "Resource potential Management", "Hydropower resource potential management } \\
\text { approaches", "Hydropower resource potential Evaluation", "Hydropower resource } \\
\text { potential development approaches" "Hydropower resource, potential development } \\
\text { techniques", "Hydropower resource potential measurement", "Resource Potential", } \\
\text { "Hydropower resource potential Analysis", "Resource potential management } \\
\text { methodologies", "Hydropower resource potential methodologies", "Resource potential } \\
\text { Essentials", "Resource potential methods", "Resource potential development processes", } \\
\text { "Resource potential practices", "Resource potential approaches", Hydropower resource } \\
\text { potential techniques". }\end{array}$ \\
\hline
\end{tabular}

\subsection{Literature Publication Search Strategy}

As hydropower resource potential is with diverse terminology so we decided to use the search string with different synonyms of hydropower resource potential such as "Sustainable energy resource", "Hydroelectricity", "Renewable energy source", "Sustainable hydropower", "Hydroelectric energy", "Hydropower technology", "Hydroelectric power "Literature published between 1993-2019 are selected to search by using only the tittleand keywords fields

\section{3 "Inclusion" and "Exclusion "Criteria of Literature}

"Inclusion" criteria include

(1) screened out the research study for resource potential trends and status "Exclusion" criteria include

(1) Eliminate research study about trends and status in other fields

(2) Excluding copy sections, barring those without total information. Eliminate studies without full text. 


\section{Data Extraction / Publication Quality Assessment}

For the information extraction the summary sheet was designed from the chosen literature. That will extricate the at finally chosen papers surveying their quality and searching answers for research questions

\section{Quality Assessment Detail 1-5:}

1. Literature provides detailed information of hydropower resource potential and resource potential management trends?

The possible responses to this question are: "Yes (a)" for the detail description of hydropower resource potential trends around the globe; "partially (b)" for the partial or not detail information about hydropower resource potential trends around the globe; and "No (c)" paper having no information about resource potential management trends

2. Literature gives the enough tenet as how the hydropower resource potential management procedures are applied across the globe?

The possible responses to this question are "Yes (a)" for having the information as how the hydro resource potential management techniques helped out across the globe; "partially (b)" paper having partial or not detail information as how the hydropower resource potential management techniques helped out across the globe; and "No (c)" paper having no information as how the hydropower resource potential techniques helped out across the globe.

3. The literature provides clear outcomes of hydropower resource potential management status for across the globe?

The possible responses to this question are: "Yes (a)" paper having clear outcomes; "partially (b) " for paper having partial or not detail results; and "No (c)" for paper having no outcomes.

\section{The literature has been published in a relevant journal or conference proceedings?}

The possible responses to this question are: "Very relevant (a)"; "Relevant (b)", and "Not so relevant (c)" . This question will be evaluated by considering the order of significance provided by the computerized library, the CORE conference positioning (A, B and C conferences), and the Journal Citation Reports (JCR) lists.

\section{The study has been cited by other authors?}

The possible responses to this question are: "Yes (a)" on the off chance that the paper has been cited by more than five creators; "partially (b)" in case the paper has been cited by 1 to 5 (1-5) authors; and "No (c)" for no citation. This question was appraised by considering the Google researcher citations count 
Electrical Engineering: An International Journal (EEIJ) Vol.7, No.1/2, June 2020

Table 4: Data Extraction and Publication Quality Assessment

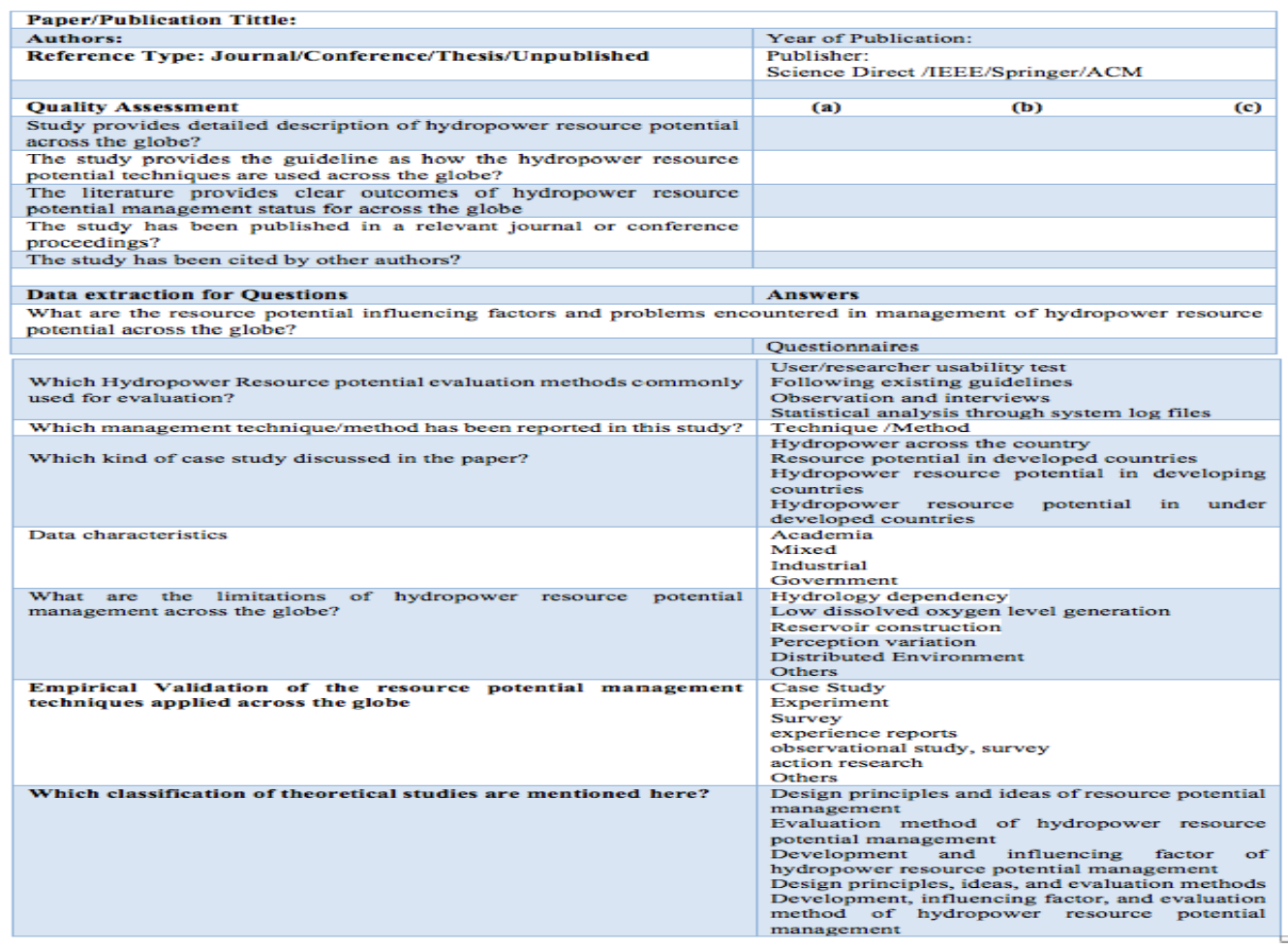

\section{Publication's General Information}

Table 5 shows the general details of publications within the systematic study audit prepare all these papers were finalized to gather the information according to our designed questions.

Through searching of the literature for 27 years within the four databases, we got collective of 8035 published studies. After implementation of our "inclusion" and "exclusion" criteria, we considered 61 publications for analysis

\section{Reasons for the high exclusion rate are as follows.}

1) It happens commonly that repetition of same publication occurs in databases.

2) Unrelated field of study retrieved commonly.

3) Search function limitation by databases

Table 5: Information Regarding Databases Literature

\begin{tabular}{|l|l|l|l|l|l|}
\hline S.No & Information Regarding Databases Literature & $\begin{array}{l}\text { Journal/ } \\
\text { Database } \\
\text { (Digital } \\
\text { Library) }\end{array}$ & $\begin{array}{l}\text { Yonference/Chapter/ } \\
\text { Magazine }\end{array}$ & Year \\
\hline 1 & Tittle & $\begin{array}{l}\text { Renewable energy } \\
\text { deployment to combat } \\
\text { energy crisis in Pakistan }\end{array}$ & $\begin{array}{l}\text { Abdul Raheem, } \\
\text { Sikandar Ali Abbasi, } \\
\text { Asif Memon, Saleem } \\
\text { R. Samo, Y. H. } \\
\text { Taufiq-Yap, Michael } \\
\text { K. } \\
\text { Danquah and Razif } \\
\text { Harun }\end{array}$ & & 2016 \\
& & & & \\
\hline
\end{tabular}


Electrical Engineering: An International Journal (EEIJ) Vol.7, No.1/2, June 2020

\begin{tabular}{|c|c|c|c|c|c|}
\hline 02 & $\begin{array}{l}\text { India-Pakistan Energy } \\
\text { Cooperation: Rethinking } \\
\text { Opportunities and Newer } \\
\text { Approaches }\end{array}$ & Mahendra P. La ma & Springer & Chapter & 2014 \\
\hline 03 & $\begin{array}{l}\text { Global Expansion of } \\
\text { Renewable Energy } \\
\text { Generation: An Analysis } \\
\text { of Policy Instruments }\end{array}$ & $\begin{array}{l}\text { Sanya Carley, } \\
\text { Elizabeth Baldwin } \\
\text { Lauren M. MacLe } \\
\text { an, } \\
\text { Jennifer N. Brass }\end{array}$ & Springer & Journal & 2017 \\
\hline 04 & \begin{tabular}{|l|} 
The role of renewable \\
and non- renewable \\
energy consumption in \\
$\mathrm{CO}_{2}$ emissions: a \\
disaggregate analysis of \\
Pakistan
\end{tabular} & \begin{tabular}{|l|} 
Syed Anees Haide r \\
Zaidi, Danish, Fujun \\
Hou, \\
Faisal Mehmood \\
Mirza
\end{tabular} & Springer & Journal & 2018 \\
\hline 05 & \begin{tabular}{|l|} 
Solar Energy: \\
Topographical Asset for \\
Pakistan
\end{tabular} & \begin{tabular}{|l|} 
Pervez Hameed \\
Shaikh, Faheemullah \\
Shaikh, and Mushtaq \\
Mirani
\end{tabular} & Springer & Journal & 2013 \\
\hline 06 & $\begin{array}{l}\text { Potential consequences } \\
\text { of projected climate } \\
\text { change impacts on } \\
\text { hydroelectricity } \\
\text { generation }\end{array}$ & Pierre Mukheibir & Springer & Journal & 2013 \\
\hline 07 & $\begin{array}{l}\text { Poverty, environment } \\
\text { and economic growth: } \\
\text { exploring the links } \\
\text { among three complex } \\
\text { issues with specific } \\
\text { focus on the Pakistan's } \\
\text { case }\end{array}$ & Himayatullah Khan & Springer & Journal & 2008 \\
\hline 08 & $\begin{array}{l}\text { An econometric analysis } \\
\text { of inter-fuel substitution } \\
\text { in energy } \\
\text { sector of Pakistan }\end{array}$ & $\begin{array}{l}\text { Waqar Khalid, } \\
\text { Abdul Jalil }\end{array}$ & Springer & Journal & 2019 \\
\hline 09 & $\begin{array}{l}\text { The Future of Energy } \\
\text { II: Renewable Energy }\end{array}$ & Francis F. Chen & Springer & Chapter & 2011 \\
\hline 10 & $\begin{array}{l}\text { Evaluating renewable } \\
\text { energy sources for } \\
\text { implementing the } \\
\text { hydrogen economy in } \\
\text { Pakistan: a two-stage } \\
\text { fuzzy MCDM } \\
\text { approach }\end{array}$ & \begin{tabular}{|l|} 
Li Xu, Syed Ahsan \\
Ali Shah, Hashim \\
Zameer, Yasir \\
,Ahmed Solangi
\end{tabular} & Springer & Journal & 2019 \\
\hline 11 & $\begin{array}{l}\text { Developing High- } \\
\text { Resolution Remote } \\
\text { Sensing Technology }\end{array}$ & \begin{tabular}{|l|} 
Kabiyeva Marzha n \\
Kaskina Dina
\end{tabular} & Springer & Chapter & 2018 \\
\hline
\end{tabular}


Electrical Engineering: An International Journal (EEIJ) Vol.7, No.1/2, June 2020

\begin{tabular}{|c|c|c|c|c|c|}
\hline & $\begin{array}{l}\text { into an Advanced } \\
\text { Knowledge Management } \\
\text { System to Assess Small- } \\
\text { Scale Hydropower } \\
\text { Potential } \\
\text { in Kazakhstan }\end{array}$ & Bradshaw Roland & & & \\
\hline 12 & \begin{tabular}{|l|} 
Modelling Water \\
Resource Allocation: A \\
Case Study on \\
Agriculture Versus \\
Hydropower \\
Production
\end{tabular} & $\begin{array}{l}\text { Jorge Bielsa Rosa } \\
\text { Duarte }\end{array}$ & Springer & Chapter & 2002 \\
\hline 13 & $\begin{array}{l}\text { Study on Corrosion } \\
\text { Status and Control } \\
\text { Strategies in Energy } \\
\text { Field in China }\end{array}$ & Jianyun Zhang & Springer & Chapter & 2019 \\
\hline 14 & $\begin{array}{l}\text { Applying rough random } \\
\text { MODM model to } \\
\text { resource- constrained } \\
\text { project scheduling } \\
\text { problem: A case study } \\
\text { of Pubugou } \\
\text { Hydropower Project in } \\
\text { China }\end{array}$ & $\begin{array}{l}\text { ZheZhang, Jiupin g } \\
\text { Xu }\end{array}$ & Springer & Journal & 2014 \\
\hline 15 & Hydropower & $\begin{array}{l}\text { Jingsheng Jia,Petr as } \\
\text { Punys } \\
\text { Jing Ma }\end{array}$ & Springer & Chapter & 2012 \\
\hline 16 & \begin{tabular}{|l|} 
Small Hydropower \\
Resources And \\
Prospects Of Small \\
Hydropower Electric \\
Plants In The Near- \\
Border Regions Of \\
Ukraine \\
\end{tabular} & Igor Winkler & Springer & Conference & 2009 \\
\hline 17 & $\begin{array}{l}\text { Geospatial and } \\
\text { hydrological modeling to } \\
\text { assess hydropower } \\
\text { potential zones and site } \\
\text { location over rainfall } \\
\text { dependent } \\
\text { Inland catchment }\end{array}$ & $\begin{array}{l}\text { Manish Kumar G } \\
\text { oya, Vishal Singh, } \\
\text { Akshay H. Meena }\end{array}$ & Springer & Journal & 2015 \\
\hline 18 & $\begin{array}{l}\text { Utilization of the world's } \\
\text { potential water resources } \\
\text { by hydropower } \\
\text { installations }\end{array}$ & $\begin{array}{l}\text { G. A. Pretro } \\
\text { M. P. Fedorov }\end{array}$ & Springer & Journal & 1993 \\
\hline 19 & \begin{tabular}{|l|} 
Adaptation to Climate \\
Change in the \\
Management of a \\
Canadian Water- \\
Resources System \\
Exploited for \\
Hydropower
\end{tabular} & $\begin{array}{l}\text { Marie Minville } \\
\text { François Brissette } \\
\text { Stéphane Krau } \\
\text { Robert Leconte }\end{array}$ & Springer & Journal & 2009 \\
\hline
\end{tabular}


Electrical Engineering: An International Journal (EEIJ) Vol.7, No.1/2, June 2020

\begin{tabular}{|c|c|c|c|c|c|}
\hline 20 & $\begin{array}{l}\text { Hydropower for } \\
\text { sustainable water and } \\
\text { energy development in } \\
\text { Ethiopia }\end{array}$ & \begin{tabular}{|l|} 
Dagmawi Muluge \\
ta Degefu Weijun He \\
Jian Hua Zhao
\end{tabular} & Springer & Journal & 2015 \\
\hline 21 & $\begin{array}{l}\text { The Current Situation } \\
\text { and Perspectives on the } \\
\text { Use of Hydropower for } \\
\text { Electricity } \\
\text { Generation }\end{array}$ & $\begin{array}{l}\text { Jorge Morales } \\
\text { Pedraza }\end{array}$ & Springer & Journal & 2015 \\
\hline 22 & $\begin{array}{l}\text { Harnessing Renewable } \\
\text { Energy Technologies for } \\
\text { ICT and e-Governance } \\
\text { Services in Un- } \\
\text { Electrified } \\
\text { Communities in Rural } \\
\text { Nepal }\end{array}$ & Mona Sharma & $\mathrm{ACM}$ & Conference & 2012 \\
\hline 23 & $\begin{array}{l}\text { Parasol and } \\
\text { GreenSwitch: Managing } \\
\text { Datacenters Powered by } \\
\text { Renewable Energy }\end{array}$ & $\begin{array}{l}\text { In igo Goiri, William } \\
\text { Katsak, Kien Le }{ }^{\dagger}, \\
\text { Thu D. } \\
\text { Nguyen, Ricardo } \\
\text { Bianchini }\end{array}$ & $\mathrm{ACM}$ & Conference & 2013 \\
\hline 24 & $\begin{array}{l}\text { Potential to reduce } \\
\text { energy consumption and } \\
\text { GHG emissions by using } \\
\text { renewable energy } \\
\text { technologies in the } \\
\text { conversion of existing } \\
\text { houses into net-zero and } \\
\text { near net- zero energy } \\
\text { buildings }\end{array}$ & \begin{tabular}{|l} 
S. Rasoul Asaee, \\
V. Ismet Ugursal \\
\end{tabular} & $\mathrm{ACM}$ & Conference & 2018 \\
\hline 25 & $\begin{array}{l}\text { Application of Data } \\
\text { Science for Controlling } \\
\text { Energy Crises: A Case } \\
\text { Study } \\
\text { of Pakistan } \\
\end{array}$ & $\begin{array}{l}\text { Saif Ullah, } \\
\text { Muhammad Asif, } \\
\text { Shahbaz Ahmad, } \\
\text { Ulfat Imdad, } \\
\text { Osama Sohaib } \\
\end{array}$ & ACM & Conference & 2019 \\
\hline 26 & $\begin{array}{l}\text { Leveraging Renewable } \\
\text { Energy in } \\
\text { Data Centers: Present } \\
\text { and Future }\end{array}$ & Ricardo Bianchini & $\mathrm{ACM}$ & Conference & 2012 \\
\hline 27 & $\begin{array}{l}\text { The Case for Efficient } \\
\text { Renewable Energy } \\
\text { Management in Smart } \\
\text { Homes }\end{array}$ & $\begin{array}{l}\text { Ting Zhu ,A ditya } \\
\text { Mishra, David Irwin, } \\
\text { Navin Sharma, } \\
\text { Prashant Shenoy, } \\
\text { Don } \\
\text { Towsley }\end{array}$ & ACM & Workshop & 2011 \\
\hline 28 & $\begin{array}{l}\text { Increasing Data Centre } \\
\text { Renewable Power Share } \\
\text { via Intelligent Smart } \\
\text { City } \\
\text { Power Control }\end{array}$ & $\begin{array}{l}\text { Florian Niedermeier, } \\
\text { Wolfgang Duschl } \\
\text { Torben Möller, } \\
\text { Hermann de Meer }\end{array}$ & $\mathrm{ACM}$ & Conference & 2015 \\
\hline
\end{tabular}


Electrical Engineering: An International Journal (EEIJ) Vol.7, No.1/2, June 2020

\begin{tabular}{|c|c|c|c|c|c|}
\hline 29 & $\begin{array}{l}\text { HydroNode: An } \\
\text { Underwater Sensor Nod } \\
\text { Prototype for Monitorin } \\
\text { Hydroelectric Reservoir }\end{array}$ & $\begin{array}{l}\text { Luiz F. M. Vieira, } \\
\text { David Pinto, } \\
\text { Sadraque S. Viana } \\
\text { Marcos A. M. } \\
\text { Vieira, José } \\
\text { Augusto M. Nacif, } \\
\text { Alex B. Vieira }\end{array}$ & $\mathrm{ACM}$ & Conference & 2012 \\
\hline 30 & $\begin{array}{l}\text { Energy Generation } \\
\text { Capacity Analysis of a } \\
\text { Canal Based Hydro } \\
\text { Project }\end{array}$ & $\begin{array}{l}\text { Farhan Khan , Kinza } \\
\text { Ali, Ahmed Kausar, } \\
\text { Shafaq Kausar }\end{array}$ & IEEE & Conference & 2013 \\
\hline 31 & $\begin{array}{l}\text { Energy outlook in } \\
\text { Pakistan }\end{array}$ & \begin{tabular}{|l|} 
Khuram Pervez \\
Amber, Naila \\
Ashraf
\end{tabular} & IEEE & Conference & 2014 \\
\hline 32 & $\begin{array}{l}\text { Cost Optimization of an } \\
\text { Off-Grid Hybrid } \\
\text { Renewable Energy } \\
\text { System with Battery } \\
\text { Storage for Rural } \\
\text { Electrification in } \\
\text { Pakistan } \\
\end{array}$ & \begin{tabular}{|l|} 
Rizwan Kamal, \\
Muhammad Younas, \\
Muhammad Shoaib \\
Khalid, Affaq Qamar \\
\\
\end{tabular} & IEEE & Conference & 2018 \\
\hline 33 & $\begin{array}{l}\text { Economic Evaluation of } \\
\text { Tarbela Dam }\end{array}$ & \begin{tabular}{|l|} 
Haris Mushtaq, Dr. \\
Mohammad Bilal \\
Khan, Hafeez \\
Rehman Khan, \\
Muhammad Ali \\
Zahoor
\end{tabular} & IEEE & Conference & 2015 \\
\hline 34 & $\begin{array}{l}\text { A Micro Hydro Power } \\
\text { Plant for Distributed } \\
\text { Generation using } \\
\text { Municipal Water Waste } \\
\text { with Archimedes Screw }\end{array}$ & $\begin{array}{l}\text { Ali Raza, } \\
\text { Muhammad Saleem } \\
\text { Mian, Dianguo Xu, } \\
\text { Jawad Ahmed }\end{array}$ & IEEE & Conference & 2013 \\
\hline 35 & $\begin{array}{l}\text { Optimization of Daily } \\
\text { Operation of Micro } \\
\text { Hydro Power Plant } \\
\text { Coupled with Compress } \\
\text { Air Storage }\end{array}$ & $\begin{array}{l}\text { Usama Bin Irshad, } \\
\text { M.S Javaid, } \\
\text { Saifullah Shafiq, Md } \\
\text { Shafiul Alam, } \\
\text { M.A Abido, Tahir } \\
\text { Mumtaz }\end{array}$ & IEEE & Conference & 2016 \\
\hline 36 & $\begin{array}{l}\text { Extenuating Shortfall of } \\
\text { Electric Power through } \\
\text { Potential } \\
\text { Accessible in Pakistan }\end{array}$ & $\begin{array}{l}\text { Mariyam Arif, Ye } \\
\text { Liu and Israr ul Haq }\end{array}$ & IEEE & Conference & 2018 \\
\hline 37 & $\begin{array}{l}\text { Grid Interconnection of } \\
\text { Micro Hydro Power } \\
\text { Plants: Major } \\
\text { Requirements, Key } \\
\text { Issues and Challenges }\end{array}$ & $\begin{array}{l}\text { Waqas Ali, Haroon } \\
\text { Farooq, Ata Ur } \\
\text { Rehman, Mohsin } \\
\text { Jamil, Qasim Awais } \\
\text {, Mohsin Ali }\end{array}$ & IEEE & Conference & 2018 \\
\hline
\end{tabular}


Electrical Engineering: An International Journal (EEIJ) Vol.7, No.1/2, June 2020

\begin{tabular}{|c|c|c|c|c|c|}
\hline 38 & $\begin{array}{l}\text { A predictive pan- } \\
\text { European economic and } \\
\text { production dispatch } \\
\text { model for the energy } \\
\text { transition in } \\
\text { the electricity sector }\end{array}$ & $\begin{array}{l}\text { Laurent Pagnier, } \\
\text { Philippe Jacquod }\end{array}$ & IEEE & Conference & 2017 \\
\hline 39 & $\begin{array}{l}\text { Thermal Unit } \\
\text { Commitment } \\
\text { considering Pumped } \\
\text { Storage Hydro } \\
\text { Electricity Plants }\end{array}$ & $\begin{array}{l}\text { Mary Prasanna T, } \\
\text { C.H. Ram } \\
\text { jethmalani, Dr.Sishaj } \\
\text { P Simon }\end{array}$ & iEEE & Conference & 2013 \\
\hline 40 & $\begin{array}{l}\text { Optimization of } \\
\text { Capacity and } \\
\text { Operational Scheduling } \\
\text { for Grid- Tied Microgrid } \\
\text { using Pumped-Storage } \\
\text { Hydroelectricity and } \\
\text { Photovoltaic }\end{array}$ & $\begin{array}{l}\text { Petrus Yuri Nugraha, } \\
\text { Augie Widyotriatmo } \\
\text {, Sutanto } \\
\text { Hadisupadmo, } \\
\text { Deddy Kurniadi }\end{array}$ & IEEE & Conference & 2015 \\
\hline 41 & $\begin{array}{l}\text { Interaction between } \\
\text { short-term and seasonal } \\
\text { storages in a } \\
\text { predominantly } \\
\text { renewable power } \\
\text { system }\end{array}$ & $\begin{array}{l}\text { Christoph Groiss, } \\
\text { Walter Schaffer, } \\
\text { Wolfgang Gawlik }\end{array}$ & IEEE & Conference & 2017 \\
\hline 42 & $\begin{array}{l}\text { Developing and } \\
\text { utilization } \\
\text { of hydroelectric powe r } \\
\text { and improving } \\
\text { atmospheric } \\
\text { environment }\end{array}$ & $\begin{array}{l}\text { Maoyu Ran ; Yan } \\
\text { Hu }\end{array}$ & IEEE & Conference & 2011 \\
\hline 43 & $\begin{array}{l}\text { Restless Waters: Fossil } \\
\text { Fuel Emissions } \\
\text { Conditioning a } \\
\text { Reduction } \\
\text { inHydroelectric Reso } \\
\text { urces in Chile }\end{array}$ & $\begin{array}{l}\text { Hugh } \\
\text { Rudnick ; Rodrig } \\
\text { Palma- } \\
\text { Behnke ; Andrea } \\
\text { Rudnick ; Carlos } \\
\text { Benavides }\end{array}$ & IEEE & Magazines & 2014 \\
\hline 44 & $\begin{array}{l}\text { Energy Scenario } \\
\text { and Potential of Hydr } \\
\text { oelectric Power in } \\
\text { Pakistan }\end{array}$ & \begin{tabular}{l|} 
Waqar \\
Uddin ; Sadam \\
Hussain ; Kamran \\
Zeb ; Musaib Aleem \\
Dildar ; Z. Ullah ; \\
Ihsan Ullah Khalil ; \\
R. Ullah ; A. \\
Haider ; Muhamm ad \\
Adil ; H. J. Kim
\end{tabular} & IEEE & Conference & 2018 \\
\hline 45 & $\begin{array}{l}\text { Assessment of } \\
\text { renewable energy } \\
\text { resources and the use of } \\
\text { hydro power for } \\
\text { fluctuation } \\
\text { compensation in } \\
\text { Cameroon }\end{array}$ & $\begin{array}{l}\text { M. Pendieu Kwaye ; } \\
\text { J. Bendfeld ; N. } \\
\text { Anglani, }\end{array}$ & IEEE & Conference & 2015 \\
\hline
\end{tabular}


Electrical Engineering: An International Journal (EEIJ) Vol.7, No.1/2, June 2020

\begin{tabular}{|c|c|c|c|c|c|}
\hline 46 & $\begin{array}{l}\text { A review of current } \\
\text { renewable energy } \\
\text { activities in Bangladesh }\end{array}$ & $\begin{array}{l}\text { Alimul Haque Khan } \\
\text { Kazi Rehnuma } \\
\text { Zafreen, Mir } \\
\text { Muntasir Hossain, } \\
\text { Maidul Islam, } \\
\end{array}$ & IEEE & Conference & 2015 \\
\hline 47 & $\begin{array}{l}\text { Prospects of rural } \\
\text { electrification of } \\
\text { Balochistan province } \\
\text { with renewable energy } \\
\text { sources }\end{array}$ & $\begin{array}{l}\text { Anis Ur Rehman ; } \\
\text { Syed Mushtaq A. } \\
\text { Shah ; Syed Ali } \\
\text { Raza Shah ; Saeed } \\
\text { Badshah ; M.A. } \\
\text { Khattak, }\end{array}$ & IEEE & Conference & 2017 \\
\hline 48 & $\begin{array}{l}\text { Feasibility and } \\
\text { simulation study of high } \\
\text { rise building Micro-grid } \\
\text { with PV } \\
\text { and mini-hydro pumping }\end{array}$ & $\begin{array}{l}\text { Jianmin } \\
\text { Zhang ; Qianzhi } \\
\text { Zhang, }\end{array}$ & IEEE & Conference & 2013 \\
\hline 49 & $\begin{array}{l}\text { Optimal Electric Energy } \\
\text { Production scheduling } \\
\text { for Thermal-Hydro } \\
\text { Electric Power } \\
\text { Systems }\end{array}$ & Jiekang Wu, & IEEE & Conference & 2009 \\
\hline 50 & \begin{tabular}{|l|} 
Interface model based \\
cyber-physical energy \\
system design for smart \\
grid
\end{tabular} & $\begin{array}{l}\text { Janet } \\
\text { Roveda ; Susan } \\
\text { Lysecky ; Young- } \\
\text { Jun } \\
\text { Son ; Hyungtaek } \\
\text { Chang; Anita } \\
\text { Annamalai } \\
\end{array}$ & IEEE & Conference & 2011 \\
\hline 51 & $\begin{array}{l}\text { "Water resources } \\
\text { planning hydropower for } \\
\text { sustainable and green } \\
\text { energy in } \\
\text { Turkey }\end{array}$ & $\begin{array}{l}\text { Ibrahim Yuksel ; } \\
\text { Hasan } \\
\text { Arman ; Ibrahim } \\
\text { Halil Demirel }\end{array}$ & IEEE & Conference & 2018 \\
\hline 52 & $\begin{array}{l}\text { Analysis of ways of } \\
\text { solving the problem of } \\
\text { hybrid energy complexes } \\
\text { based on reserve for } \\
\text { power supply of } \\
\text { autonomous rural } \\
\text { consumers in } \\
\text { Myanmar" }\end{array}$ & $\begin{array}{l}\text { Michael G. } \\
\text { Tyagunov; Thu } \\
\text { Yein Min }\end{array}$ & IEEE & Conference & 2018 \\
\hline 53 & $\begin{array}{l}\text { "“24 years postgraduate } \\
\text { program renewable } \\
\text { energy" }\end{array}$ & $\begin{array}{l}\text { Evelyn } \\
\text { Brudler ; Michel } \\
\text { Golba ; Andreas } \\
\text { Günther ; Hans } \\
\text { Holtorf, Leonie } \\
\text { Ibing, Edu Knagge, } \\
\text { Udo } \\
\text { Kulschewski }\end{array}$ & & & 2012 \\
\hline 54 & \begin{tabular}{|l|} 
Assessment of \\
Hydropower Plants \\
Energy Production Cost \\
Influenced by
\end{tabular} & $\begin{array}{l}\text { Ionut Bogdan } \\
\text { Stoenescu ; Sorin a } \\
\text { Costinas ; Gheorg }\end{array}$ & IEEE & Conference & 2019 \\
\hline
\end{tabular}


Electrical Engineering: An International Journal (EEIJ) Vol.7, No.1/2, June 2020

\begin{tabular}{|c|c|c|c|c|c|}
\hline & \begin{tabular}{|l|} 
Operational Decisions \\
and Control Strategy
\end{tabular} & $\begin{array}{l}\text { he Marius } \\
\text { Deaconu“" }\end{array}$ & & & \\
\hline 55 & $\begin{array}{l}\text { Flow regime aspects in } \\
\text { determining } \\
\text { environmental flows and } \\
\text { maximising energy } \\
\text { production at run-of- } \\
\text { river hydropower plan ts }\end{array}$ & $\begin{array}{l}\text { Alban Kuriqi, } \\
\text { António N. Pinheiro, } \\
\text { Alvaro Sordo-Ward, } \\
\text { Luis Garrote }\end{array}$ & $\begin{array}{l}\text { Science } \\
\text { Direct }\end{array}$ & Journal & 2019 \\
\hline 56 & $\begin{array}{l}\text { Is } \\
\text { the hydropower boom } \\
\text { actually taking place? A } \\
\text { case study of a South } \\
\text { East European country, } \\
\text { Bosnia and Herzegovina }\end{array}$ & $\begin{array}{l}\text { Özge Can Dogmus, } \\
\text { Jonas } \emptyset . \text { Nielsen }\end{array}$ & $\begin{array}{l}\text { Science } \\
\text { Direct }\end{array}$ & Journal & 2019 \\
\hline 57 & $\begin{array}{l}\text { Trans-regional } \\
\text { transmission of large- } \\
\text { scale hydropower: } \\
\text { problems and solutions } \\
\text { in receiving power grid }\end{array}$ & $\begin{array}{l}\text { Jianyu } \\
\text { Lu, Jianjian Shen, } \\
\text { Chengguo Su, } \\
\text { Qianqian Shen }\end{array}$ & $\begin{array}{l}\text { Science } \\
\text { Direct }\end{array}$ & Journal & 2019 \\
\hline 58 & $\begin{array}{l}\text { The energy injustice of } \\
\text { hydropower: } \\
\text { Development, } \\
\text { resettlement, and social } \\
\text { exclusion at the } \\
\text { Hongjiang and Wanmipo } \\
\text { hydropowe r stations in } \\
\text { China }\end{array}$ & $\begin{array}{l}\text { Xiaofan Zhao, Liang } \\
\text { Wu, } \\
\text { Ye Qi }\end{array}$ & $\begin{array}{l}\text { Science } \\
\text { Direct }\end{array}$ & Journal & 2019 \\
\hline 59 & $\begin{array}{l}\text { The Hydropower Pote } \\
\text { ntial Assessment Tool } \\
\text { (HPAT): Evaluation of } \\
\text { run-of- riverresource } \\
\text { potentia } 1 \text { for any global } \\
\text { land area and application } \\
\text { to Falls Creek, Oregon, } \\
\text { USA }\end{array}$ & $\begin{array}{l}\text { Thomas M. Mosier, } \\
\text { Kendra V. Sharp, } \\
\text { David F. Hill } \\
\end{array}$ & $\begin{array}{l}\text { Science } \\
\text { Direct }\end{array}$ & Journal & 2016 \\
\hline 60 & $\begin{array}{l}\text { Resource assessment and } \\
\text { feasibility study for the } \\
\text { generation of } \\
\text { hydrokinetic power in } \\
\text { the tailwaters of selected } \\
\text { hydropower s tations in } \\
\text { Nigeria }\end{array}$ & $\begin{array}{l}\text { Laniyi L. } \\
\text { Ladokun,Bolaji F. } \\
\text { Sule, Kajogbola } \\
\text { R. Ajao, Adeniyi } \\
\text { G. Adeogun }\end{array}$ & Science & Journal & 2018 \\
\hline 61 & $\begin{array}{l}\text { Temporal analysis of } \\
\text { water-energy nexus } \\
\text { indicators } \\
\text { for hydropower gener } \\
\text { ation and water }\end{array}$ & $\begin{array}{l}\text { Mohammed } \\
\text { Basheer,Nadir } \\
\text { Ahmed Elagib }\end{array}$ & $\begin{array}{l}\text { Science } \\
\text { Direct }\end{array}$ & Journal & 2019 \\
\hline
\end{tabular}


Electrical Engineering: An International Journal (EEIJ) Vol.7, No.1/2, June 2020

\begin{tabular}{|l|l|l|l|l|}
\hline $\begin{array}{l}\text { pumping in the Lower } \\
\text { Blue Nile Basin }\end{array}$ & & & & \\
\hline
\end{tabular}

$[8,9,10,11,12,13,14,15, ` 16,17,18,19,20,21,22,23,24,25,26,27,28,29,30,31,32,33,34,35,36,37,38$, $39,40,41,42,43,44 ., 45,46,47,48,49,50,51,52,53,54,55,56,57,58,59,60,61,62,63,64,65,66,67,68]$

\section{Graphical depiction of analysed data outcomes and Specific Information related to existing literature}

\begin{tabular}{|c|c|c|c|c|c|}
\hline \multicolumn{3}{|l|}{ Q5: Source of Publication } & \multicolumn{3}{|l|}{ Q6: Publisher } \\
\hline Options & Response \% & Responses & Options & Response \% & Responses \\
\hline Conference & 52.45 & 32 & Springer & 34.42 & 21 \\
\hline $\begin{array}{l}\text { Chapters } \\
\text { Joumals }\end{array}$ & $\begin{array}{l}9.83 \\
34.42\end{array}$ & $\begin{array}{l}6 \\
21\end{array}$ & $\mathrm{ACM}$ & 13.11 & 08 \\
\hline $\begin{array}{l}\text { Joumals } \\
\text { Workshop }\end{array}$ & 1.63 & 1 & IEEE & 40.98 & 25 \\
\hline Magazines & 1.63 & $i$ & Science Direct & 11.47 & 07 \\
\hline Total Responses $=61$ & Answered Responses $=61$ & Skipped $=0$ & Total Responses= & Answered Responses $=61$ & Skipped $=0$ \\
\hline Mean $=1.51$ & & & Mean $=2.393$ & & \\
\hline Standard Deviation $=0.566$ & & & Standard Deviation $=0.143$ & & \\
\hline $\begin{array}{l}\text { Vaniance }=0.321 \\
\text { Standard Eror }=0.073\end{array}$ & & & Vanance $=1.243$ & & \\
\hline Standard Error $=0.073$ & & & Standard Enor=1.115 & & \\
\hline
\end{tabular}

Figure 1: Source of Publication

Figure 2: Publisher

\begin{tabular}{|c|c|c|}
\hline \multicolumn{3}{|c|}{ Q8: Study provides detailed description of hydropower resource potential across the globe? } \\
\hline Options & Response \% & Responses \\
\hline a & 22.9 & 14 \\
\hline b & 77.04 & 47 \\
\hline c & 0 & 0 \\
\hline Total Responses $=61$ & Answered Responses $=61$ & Skipped $=0$ \\
\hline \multicolumn{3}{|l|}{ Mean $=0.770$} \\
\hline \multicolumn{3}{|l|}{ Standard Deviation $=0.424$} \\
\hline \multicolumn{3}{|l|}{ Variance $=0.179$} \\
\hline Standard Eror $=0.054$ & & \\
\hline
\end{tabular}

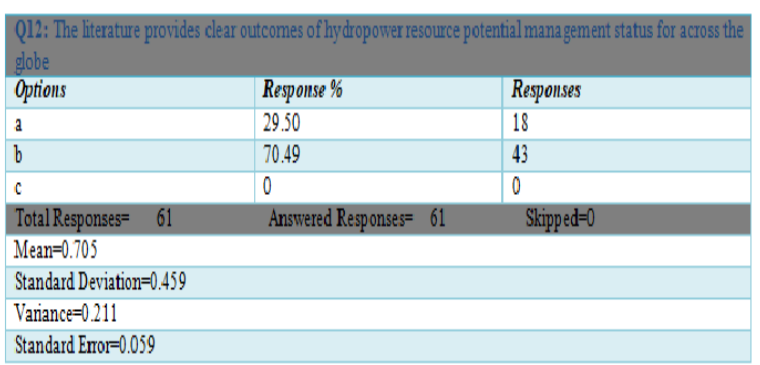

Figure 3: Study provides detailed description of hydropower resource potential across the globe

Figure 4: The literature provides clear outcomes of hydropower resource potential management status for across the globe

\begin{tabular}{|c|c|c|}
\hline Options & Response \% & Respolises \\
\hline a & 93.44 & 57 \\
\hline$b$ & 6.55 & 4 \\
\hline c & 0 & 0 \\
\hline Total Responses $=\quad 61$ & Answered Responses= 61 & Skipped $=0$ \\
\hline \multicolumn{3}{|l|}{ Mean $=0.9344262$} \\
\hline \multicolumn{3}{|l|}{ Standard Deviation $=0.2495898$} \\
\hline \multicolumn{3}{|l|}{ Vaniance $=0.6229508$} \\
\hline Standard Emol=0.0319567 & & \\
\hline
\end{tabular}

\begin{tabular}{|l|l|l|}
\hline Q14: The study has been published in a relevant joumal or conference proceedings \\
\hline Options & Response \% & Responses \\
\hline a & 19.67 & 12 \\
\hline b & 80.3 & 49 \\
\hline c & 0 & 0 \\
\hline Total Responses $=61$ & Answered Responses= 61 & Skipped $=0$ \\
\hline Mean $=0.803$ & & \\
\hline Standard Deviation $=0.401$ & & \\
\hline Vaniance $=0.161$ & & \\
\hline Standard Enor $=0.051$ & & \\
\hline
\end{tabular}

Figure 6: The study has been cited by other authors

Figure 5: The study has been published in a relevant journal or conference proceedings

\section{Conclusion}

In this systematic review (SR) performed on resource potential management and hydropower resource potential around the globe, three research questions were established and research strings were designed using PICOC structure to extract research papers from different database, including ACM, IEEE, Springer, and Science Direct. Search protocol was designed for setting studies rules regulations to follow for summarize and concrete results after analysis. 
On the basis of set protocols how the resource potential techniques are used around the globe for handling the hydropower resources while only 25 percent studies partially describe the techniques applications. In SR 75 percent study provides clear results of resource potential management status across the world, 25 percent are partially providing results of applications.

From these results, we found that so many researchers are doing studies on hydropower management. Their aim is to find out the existing constraints. They are also working on remedial techniques for management engineering for the hydropower. The aim of this study is to perform the active deep analysis of hydropower resource potential study across the globe Papers were chosen stress perceptive against every analysis question from these finalized databases 61 paper were chosen, these selected papers were analysed, assessed associate degree a based mostly by knowledge extraction was performed according to data extraction form . The collected data is statistically analysed by Statistical software "Spss" and according to this analysis, research papers selected for this study were taken between 1993-2019, out of 100 percent $34 \%$ papers were published in journal $52 \%$ in conference and $10 \%$ chapters and 2\% workshops and 2\% magazines publications.

\section{REFERENCES}

1. S. C. Bhattacharyya, "Energy access programs and sustainable development: A critical review and analysis,” Energy Sustain. Dev., vol. 16, no. 3, pp. 260-271, Sept. 2012.

2. S. Malik, M. K. Hayat, and M. U. Hayat, "External debt and economic growth: Empirical evidence from Pakistan," International Research Journal of Finance and Economics, vol. 44, no. 44, pp. 14502887, 2010

3. Abdel aziz, E.A., Saidur, R., Mekhilef, S. "A review on energy saving strategies in industrial sector". Renewable and Sustainable Energy Reviews, 15, 2011, pp.150-168.

4. International Energy Association (2015) Energy and Climate Change, World Energy Outlook special report(page\#18).Retrievedon4thOctober2019from.https://www.iea.org/publications/freepublications/pu blication/ WEO2015SpecialReportonEnergyandClimateChange.pdf

5. Apergis N, Payne JE (2011a) On the causal dynamics between renewable and non-renewable energy consumption and economic growth in developed and developing countries. Energy Syst 2:299-312. https://doi.org/10.1007/s12667-011-0037 6

6. REN21 (2011) Renewables 2011 global status report. REN21 Secretariat, Paris retrieved on 6th December 2019

7. EIA (2010) International Energy Annual 2006: electricity generation. US Department of Energy. http://www.eia.gov/cfapps/ipdbproject/iedindex3.cfm?tid=6\&pid=33\&aid=12\&cid=all,\&syid=2007 \&eyid= 2011\&unit=BKWH. Retrived on 10th November 2019

8. Abdul Raheem, Sikandar Ali Abbasi, Asif Memon, Saleem R. Samo, Y. H. Taufiq-Yap, Michael K. Danquah, Razif Harun "Renewable energy deployment to combat energy crisis in Pakistan" Energy, Sustainability and Society 2016

9. Mahendra P. Lama ,"India-Pakistan Energy Cooperation: Rethinking Opportunities and Newer Approaches " 2014

10. Sanya Carley, Elizabeth Baldwin, Lauren M. MacLean, Jennifer N. Brass, "Global Expansion of Renewable Energy Generation: An Analysis of Policy Instruments "Environmental and Resource Economics , October 2017, Volume 68, Issue 2, pp 397-440

11. Syed Anees Haider Zaidi, Danish, Fujun Hou, Faisal Mehmood Mirza , "The role of renewable and non-renewable energy consumption in CO2 emissions: a disaggregate analysis of Pakistan" Environmental Science and Pollution Research, November 2018, Volume 25, Issue 31, pp 3161631629

12. Pervez Hameed Shaikh, Faheemullah Shaikh, and Mushtaq Mirani "Solar Energy: Topographical Asset for Pakistan” Applied Solar Energy ,January 2013, Volume 49, Issue 1, pp 49-53

13. Pierre Mukheibir "Potential consequences of projected climate change impacts on hydroelectricity generation, Climatic Change November 2013, Volume 121, Issue 1, pp 67-78 
Electrical Engineering: An International Journal (EEIJ) Vol.7, No.1/2, June 2020

14. Himayatullah Khan, " Poverty, environment and economic growth: exploring the links among three complex issues with specific focus on the Pakistan's case ",Environment, Development and Sustainability December 2008, Volume 10, Issue 6, pp 913-929

15. Waqar Khalid, Abdul Jalil "'An econometric analysis of inter-fuel substitution in energy sector of Pakistan” Environmental Science and Pollution Research ,June 2019, Volume 26, Issue 17, pp 1702117031

16. Francis F. Chen, "The Future of Energy II: Renewable Energy”, An Indispensable Truth pp 75175,2011

17 .Li Xu, Syed Ahsan Ali Shah, Hashim Zameer, Yasir, Ahmed Solangi, "Evaluating renewable energy sources for implementing the hydrogen economy in Pakistan: a two-stage fuzzy MCDM approach , Environmental Science and Pollution Research, November 2019, Volume 26, Issue 32, pp 3320233215,2019

18. Kabiyeva Marzhan, Kaskina Dina, Bradshaw Roland, "Developing High-Resolution Remote Sensing Technology into an Advanced Knowledge Management System to Assess Small-Scale Hydropower Potential in Kazakhstan" , Exergy for A Better Environment and Improved Sustainability 2 pp 581605,2018

19. Jorge Bielsa, Rosa Duarte, “ Modelling Water Resource Allocation: A Case Study on Agriculture Versus Hydropower Production” Economics of Sustainable Energy in Agriculture pp 157-175, 2002

20. Jianyun Zhang, "Study on Corrosion Status and Control Strategies in Energy Field in China", The Cost of Corrosion in China pp 251-554, 2019

21. Zhe Zhang, Jiuping $\mathrm{Xu}$, “Applying rough random MODM model to resource-constrained project scheduling problem: A case study of Pubugou Hydropower Project in China",

22. Jingsheng Jia, Petras Punys, Jing Ma, "Hydropower", KSCE Journal of Civil Engineering, June 2014, Volume 18, Issue 5, pp 1279-1291,2012

23. Igor Winkler, "Small Hydropower Resources And Prospects Of Small Hydropower Electric Plants In The Near-Border Regions Of Ukraine, Energy and Environmental Challenges to Security pp 371378,2009

24. Mona Sharma, "Harnessing Renewable Energy Technologies for ICT and e-Governance Services in Un-Electrified Communities in Rural Nepal" , 6th international conference on theory and practice of electronic Governace pages 365-368,2012

25. In igo Goiri, William Katsak, Kien Le†, Thu D. Nguyen, Ricardo Bianchini ,"Parasol and GreenSwitch: Managing Datacenters Powered by Renewable Energy, 18th international conference on Architectural support for programmi ng languages and operating systems ,pages 51-64,2013

26. S. Rasoul Asaee , V. Ismet Ugursal, "Potential to reduce energy consumption and GHG emissions by using renewable energy technologies in the conversion of existing houses into net-zero and near netzero energy buildings", 4th International Conference on Engineering \& MIS 2018

27. Saif Ullah, Muhammad Asif, Shahbaz Ahmad, Ulfat Imdad, Osama Sohaib , "Application of Data Science for Controlling Energy Crises: A Case Study of Pakistan " 8th International Conference on Software and Computer Applications, 2019

28. Ricardo Bianchini, "Leveraging Renewable Energy in Data Centers: Present and Future" , 21st international conference on high Performance parallel and Distributed Computing Pages 135- 136,201

29. Ting Zhu ,A ditya Mishra, David Irwin, Navin Sharma, Prashant Shenoy, Don Towsley, "The Case for Efficient Renewable Energy Management in Smart Homes" Third ACM Workshop on Embedded Sensing Systems for Energy-Efficiency in Buildings,Pages 67-72, 2011

30. Florian Niedermeier, Wolfgang Duschl, Torben Mö ller, Hermann de Meer , "Increasing Data Centre Renewable Power Share via Intelligent Smart City Power Control”, 2015 ACM Sixth International Conference on Future Energy Systems

31. Luiz F. M. Vieira, David Pinto, Sadraque S. Viana, Marcos A. M. Vieira, José Augusto M. Nacif, Alex B. Vieira , "HydroNode: An Underwater Sensor Node Prototype for Monitoring Hydroelectric Reservoirs" Seventh ACM International Conference on Underwater Networks and Systems,2012

32. Farhan Khan, Kinza Ali, Ahmed Kausar, Shafaq Kausar , "Energy Generation Capacity Analysis of a Canal Based Hydro Project", 2012 IEEE International Conference on Power Electronics, Drives and Energy Systems

33. Khuram Pervez Amber, Naila Ashraf, Energy outlook in Pakistan , 2014 International Conference on Energy Systems and Policies (ICESP)

34. Rizwan Kamal, Muhammad Younas, Muhammad Shoaib Khalid, Affaq Qamar, "Cost Optimization of an Off-Grid Hybrid Renewable Energy System with Battery Storage for Rural Electrification in Pakistan, 2018 Clemson University Power Systems Conference (PSC) 
Electrical Engineering: An International Journal (EEIJ) Vol.7, No.1/2, June 2020

35. Haris Mushtaq, Dr. Mohammad Bilal Khan, Hafeez Rehman Khan, Muhammad Ali Zahoor , “ Economic Evaluation of Tarbela Dam", 2015 Power Generation System and Renewable Energy Technologies (PGSRET)

36. Ali Raza, Muhammad Saleem Mian, Dianguo Xu, Jawad Ahmed, A Micro Hydro Power Plant for Distributed Generation using Municipal Water Waste with Archimedes Screw ,INMIC Multi Topic, IEEE International Conference,2013

37. Usama Bin Irshad, M.S Javaid, Saifullah Shafiq, Md Shafiul Alam, M.A Abido, Tahir Mumtaz,Optimization of Daily Operation of Micro Hydro Power Plant Coupled with Compress Air Storage, 2016 19th International Multi-Topic Conference (INMIC)

39. Waqas Ali , Haroon Farooq, Ata Ur Rehman, Mohsin Jamil, Qasim Awais , Mohsin Ali "Grid Interconnection of Micro Hydro Power Plants: Major Requirements, Key Issues and Challenges" , 2018 International Symposium on Recent Advances in Electrical Engineering (RAEE)

40. Laurent Pagnier, Philippe Jacquod "A predictive pan-European economic and production dispatch model for the energy transition in the electricity sector", 2017 IEEE Manchester PowerTech

41. Mary Prasanna T, C.H. Ram jethmalani, Dr.Sishaj P Simon , Thermal Unit Commitment considering Pumped Storage Hydro Electricity Plants , 2013 International Conference on Energy Efficient Technologies for Sustainability

42. Petrus Yuri Nugraha, Augie Widyotriatmo, Sutanto Hadisupadmo , Deddy Kurniadi , "Optimization of Capacity and Operational Scheduling for Grid-Tied Microgrid using Pumped- Storage Hydroelectricity and Photovoltaic , 2015 10th Asian Control Conference (ASCC)

43. Christoph Groiss, Walter Schaffer, Wolfgang Gawlik, "Interaction between short-term and seasonal storages in a predominantly renewable power system”, CIRED - Open Access Proceedings Journal , Volume: 2017, Issue: 1, 2017

44. Maoyu Ran ; Yan $\mathrm{Hu}$,Developing and utilization of hydroelectric power and improving atmospheric environment", 2011 International Conference on Electrical and Control Engineering,2011

45. Hugh Rudnick ; Rodrigo Palma-Behnke ; Andrea Rudnick ; Carlos Benavides, "Restless Waters: Fossil Fuel Emissions Conditioning a Reduction in Hydroelectric Resources in Chile, IEEE Power and Energy Magazine ,Volume: 12 , Issue: 5 , Sept.-Oct. 2014

46. Waqar Uddin ; Sadam Hussain ; Kamran Zeb ; Musaib Aleem Dildar ; Z. Ullah ; Ihsan Ullah Khalil; R. Ullah ; A. Haider ; Muhammad Adil ; H. J. Kim, Energy Scenario and Potential of Hydroelectric Power in Pakistan", 2018 International Conference on Power Generation Systems and Renewable Energy Technologies (PGSRET)

47. Manish Kumar Goya, Vishal Singh,Akshay H. Meena "Geospatial and hydrological modeling to assess hydropower potential zones and site location over rainfall dependent Inland catchment" , Water Resources Management, June 2015, Volume 29, Issue 8, pp 2875-289

48. G. A. Pretro, M. P. Fedorov, Utilization of the world's potential water resources by hydropower installations, “ Hydrotechnical Construction”August 1993, Volume 27, Issue 8, pp 435-443

49. Marie Minville, François Brissette, Stéphane Krau, Robert Leconte, "Adaptation to Climate Change in the Management of a Canadian Water-Resources System Exploited for Hydropower", Water Resources Management, November 2009, Volume 23, Issue 14, pp 2965-2986

50. Dagmawi Mulugeta Degefu, Weijun He, Jian Hua Zhao, Jian Hua Zhao "Hydropower for sustainable water and energy development in Ethiopia, "Sustainable Water Resources Management,December 2015, Volume 1, Issue 4, pp 305-314

51. Jorge Morales ,Pedraza, "The Current Situation and Perspectives on the Use of Hydropower for Electricity Generation, Electrical Energy Generation in Europe pp 93-167,2015

52. Alban Kuriqi, António N. Pinheiro, Alvaro Sordo-Ward, Luis Garrote, "Flow regime aspects in determining environmental flows and maximising energy production at run-of-river hydropower plants”, Applied Energy ,Volume 256, 15 December 2019, 113980

53. Özge Can Dogmus, Jonas Ø. Nielsen, "Is the hydropower boom actually taking place? A case study of a South East European country, Bosnia and Herzegovina" Renewable and Sustainable Energy Reviews Volume 110, August 2019, Pages 278-289

54. Jianyu Lu, Jianjian Shen, Chengguo Su, Qianqian Shen, "Trans-regional transmission of large-scale hydropower: problems and solutions in receiving power grid" Global Energy Interconnection, Volume 2, Issue 4, August 2019, Pages 342-350

55 .Xiaofan Zhao, Liang Wu, Ye Qi , "The energy injustice of hydropower: Development, resettlement, and social exclusion at the Hongjiang and Wanmipo hydropower stations in China" Energy Research \& Social Science, Volume 62, April 2020, 101366 
Electrical Engineering: An International Journal (EEIJ) Vol.7, No.1/2, June 2020

56. Thomas M. Mosier, Kendra V. Sharp, David F. Hill, "The Hydropower Potential Assessment Tool (HPAT): Evaluation of run-of-riverresource potential for any global land area and application to Falls Creek, Oregon, USA, Renewable Energy, Volume 97, November 2016, Pages 492-503

57. Laniyi L. Ladokun,Bolaji F. Sule, Kajogbola R. Ajao, Adeniyi G. Adeogun, "Resource assessment and feasibility study for the generation of hydrokinetic power in the tailwaters of selected hydropower stations in Nigeria, Water Science, Volume 32, Issue 2, October 2018, Pages 338-354

58. Mohammed Basheer,Nadir Ahmed Elagib, "Temporal analysis of water-energy nexus indicators for hydropower generation and water pumping in the Lower Blue Nile Basin", Journal of Hydrology, Volume 578, November 2019, 124085

59. M. Pendieu Kwaye ; J. Bendfeld ; N. Anglani, "Assessment of renewable energy resources and the use of hydro power for fluctuation compensation in Cameroon" 2015 5th International Youth Conference on Energy (IYCE)

60. Alimul Haque Khan, Kazi Rehnuma Zafreen, Mir Muntasir Hossain, Maidul Islam,A review of current renewable energy activities in Bangladesh, 2015 3rd International Conference on Green Energy and Technology (ICGET)

61. Anis Ur Rehman ; Syed Mushtaq A. Shah ; Syed Ali Raza Shah ; Saeed Badshah ; M.A. Khattak, "Prospects of rural electrification of Balochistan province with renewable energy sources"3rd International Conference on Power Generation Systems and Renewable Energy Technologies (PGSRET),2017

62. Jiekang $\mathrm{Wu}$, Optimal Electric Energy Production scheduling for Thermal-Hydro Electric Power Systems, 2009 Asia-Pacific Power and Energy Engineering Conference

63. Jianmin Zhang ; Qianzhi Zhang, Feasibility and simulation study of high-rise building Micro-grid with PV and mini-hydro pumping, 2013 IEEE Power \& Energy Society General Meeting

64. Janet Roveda ; Susan Lysecky ; Young-Jun Son ; Hyungtaek Chang ; Anita Annamalai "Interface model based cyber-physical energy system design for smart grid" 2011 IEEE/IFIP 19th International Conference on VLSI and System-on-Chip

65.Ibrahim Yuksel ; Hasan Arman ; Ibrahim Halil Demirel, "Water resources planning hydropower for sustainable and green energy in Turkey", 2018 5th International Conference on Renewable Energy: Generation and Applications (ICREGA)

66. Michael G. Tyagunov ; Thu Yein Min, "Analysis of ways of solving the problem of hybrid energy complexes based on reserve for power supply of autonomous rural consumers in Myanmar", 2018 Renewable Energies, Power Systems \& Green Inclusive Economy (REPS-GIE)

67. Evelyn Brudler ; Michel Golba ; Andreas Günther ; Hans Holtorf, Leonie Ibing, Edu Knagge, Udo Kulschewski, "24 years postgraduate program renewable energy" 2nd International Conference on the Developments in Renewable Energy Technology (ICDRET 2012)

68. Ionut Bogdan Stoenescu ; Sorina Costinas ; Gheorghe Marius Deaconu“Assessment of Hydropower Plants Energy Production Cost Influenced by Operational Decisions and Control Strategy”, 2019 22nd International Conference on Control Systems and Computer Science (CSCS).

\section{AUTHORS}

Qasim Rauf got his bachelor's degree BSc Electrical Engineering from Gujranwala institute of Future Technology (GIFT )Gujranwala ,Pakistan. Currently, he is doing his Master's (MS ) in Power engineering from North China University of Water Resources and Electric Power (NCWU) Henan, China. He have taken an interest voluntary in Blood Donating Society (GIFT Blood Group Society), was also the Vice President of GIFT Character Building Society. He got best presentation awards at International Conference in 2019. He is the co-author of different research papers His current research areas encompasses Power engineering \& Renewable energy.

Yanpin Li received her master's degree from the North China University of Water Resources and Electric Power. Five years later, and Ph.D. in engineering from the School of Water Resources and Hydropower, Xi'an University of Technology. Two years later, she obtained a postdoctoral degree from Jiangsu University. Now, the main research direction of Teacher $\mathrm{Li}$ is the research and development of fluid machinery for waste energy recovery. In 2015,she also undertook the National Natural Science Foundation project of " High-pressure Recovery Turbine Model Multi-stage Hydraulic Turbine Flow Path Optimization and Design Theory”.

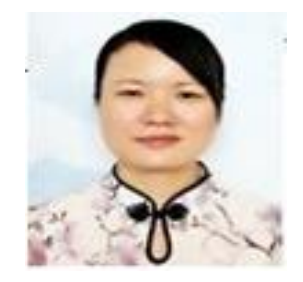


Electrical Engineering: An International Journal (EEIJ) Vol.7, No.1/2, June 2020

Anam Ashraf got her master's degree MS (SE) National University of Sciences \&Technology (NUST), Islamabad, Pakistan. Currently, she is doing PHD in Management Sciences and Engineering from North China University of Water Resources and Electric Power (NCWU) Henan, China. She has an experience of over one year in the capacity of Information Security at Ultra Spectra Pvt. Ltd, Islamabad. She also had the experience of Quality Testing and Web Designing while working as a Software Quality Test Engineer at Center for Advanced Research in Engineering (CARE) Pvt. Ltd. Moreover, she had teaching experience at multiple institutes as

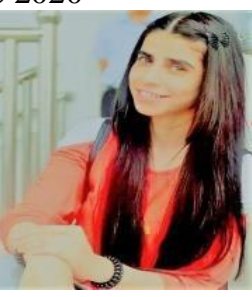
Lecturer. Her research areas encompasses Internet of Thing (IoT) Smart Home technology, Usability Engineering ,Inclusive Education Management, and Artificial Intelligence. 